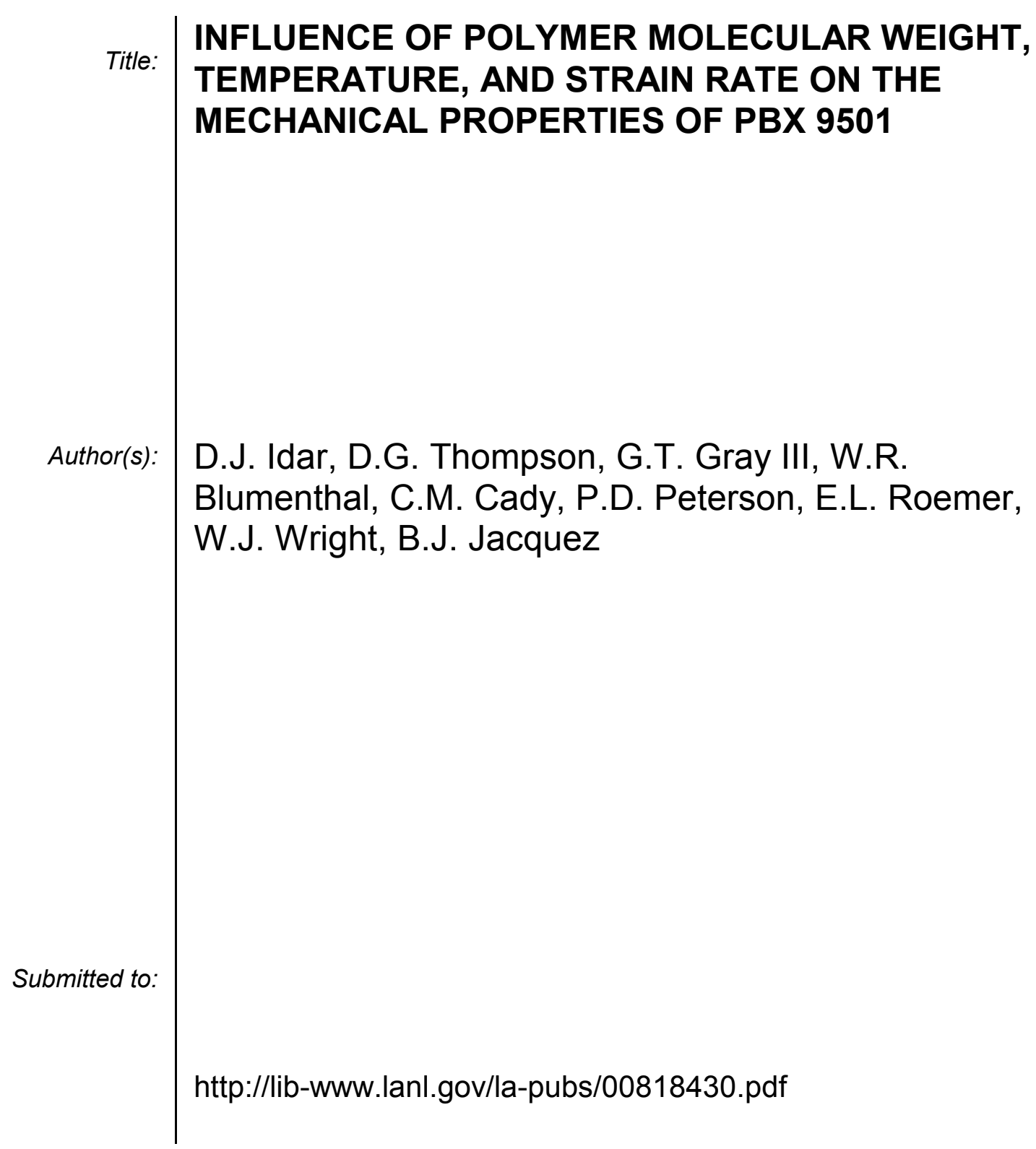




\title{
INFLUENCE OF POLYMER MOLECULAR WEIGHT, TEMPERATURE, AND STRAIN RATE ON THE MECHANICAL PROPERTIES OF PBX 9501
}

\author{
D.J. Idar, ${ }^{\dagger}$ D.G. Thompson, ${ }^{\dagger \dagger}$ G.T. Gray III, ${ }^{\dagger \dagger}$ W.R. Blumenthal, ${ }^{\dagger \dagger \dagger}$ C.M. Cady,${ }^{\dagger \dagger}$ \\ P.D. Peterson, ${ }^{\dagger \dagger}$ E.L. Roemer, ${ }^{\dagger \dagger}$ W.J. Wright,${ }^{\dagger \dagger}$ B.J. Jacquez ${ }^{\dagger \dagger \dagger}$ \\ ${ }^{\dagger}$ MS P915, ${ }^{\dagger \dagger}$ MS C920, ${ }^{\dagger \dagger}$ MS G755, Los Alamos National Laboratory, Los Alamos, NM 87545
}

\begin{abstract}
Compression and tensile measurements were conducted on newly formulated (baseline) and lower molecular weight (virtually-aged) plastic-bonded explosive PBX 9501. The PBX 9501 binder system is composed of nitroplasticized Estane $5703,{ }^{\mathrm{TM}}$ a polyester polyurethane copolymer. The molecular weight of polyester urethanes can degrade with time as a function of hydrolysis, affecting the mechanical behavior of the polymer or a polymer composite material of high explosives, i.e. PBXs. The molecular weight of Estane $5703^{\mathrm{TM}}$ was degraded by exposure to high temperature and humidity for different periods of time, and then formulated to produce "virtually-aged" PBX 9501 specimens. Quasi-static and dynamic compression tests were conducted on the baseline and virtuallyaged PBX 9501 as a function of temperature and strain rate. Quasi-static tensile tests were also conducted as a function of temperature and test rate. Rate and temperature dependence was exhibited during both compression and tensile loading. Results also show significant differences between the baseline and virtually-aged specimens for the dynamic compression tests at $-15^{\circ} \mathrm{C}$, and for the quasistatic compression tests at $-15^{\circ} \mathrm{C}, 22^{\circ} \mathrm{C}$, and $50^{\circ} \mathrm{C}$.
\end{abstract}

\section{INTRODUCTION}

Understanding and modeling the response of plastic-bonded explosives (PBXs) to low amplitude stresses and strains is critical to developing an accurate predictive capability to address potential survivability and safety issues. This requires a thorough understanding of the mechanical response of PBXs as a function of a complex set of variables including test conditions and pedigree, e.g. initial formulation conditions and changes with age. PBXs are exposed to numerous environmental conditions throughout their service lifetimes, e.g. humidity and temperature gradients, which may ultimately affect the mechanical response of these materials.

PBXs have been commonly formulated with a soft phase polymeric binder system mixed with hard phase explosive to facilitate processing, and to reduce sensitivity to different stimuli. The simplest binder matrix consists of a long-chain polymer backbone or a polymer mixed with plasticizer. Some polymeric binders are susceptible to molecular weight degradation through hydrolysis, oxidation, temperature and radiation effects.

Conventional quasi-static compression and tensile methods have been used to measure mechanical properties of PBXs at low strain rates. Dynamic compression measurements were conducted using a unique split-Hopkinson pressure bar (SHPB) designed to improve the signal-to-noise level. The new design was needed to test extremely low strength materials as compared to the maraging steel bars traditionally utilized for metals as described previously (1). The mechanical responses of PBXs have been studied as a function of strain rate and temperature as reviewed previously $(2,3)$. In general, the compressive strength and the loading modulus of polymeric materials increases with decreasing temperature and increasing strain rate with further dependence on the specimen geometry, material processing, and test method.

In the present study, we evaluate the effects of changing the Estane $5703^{\mathrm{TM}}$ (hereafter referred to as Estane) molecular weight on PBX 9501 mechanical 
properties. Quasi-static uniaxial compression and tensile tests were performed on baseline PBX 9501 and 3 different lots of virtually-aged PBX 9501 formulated from degraded Estane. Dynamic compression data on the same materials were also conducted. Data are compared as a function of the Estane molecular weight differences.

\section{EXPERIMENTAL TECHNIQUES}

\section{Materials and Preparation}

This investigation was performed on the plasticbonded explosive PBX 9501. PBX 9501 is a formulation composed of 94.9/2.5/2.5/0.1 wt\% HMX / Estane /a eutectic mixture of bis(2,2 dinitropropyl) acetal and bis (2,2-dinitropropyl) formal [abbreviated BDNPA-F] / and Irganox (a free radical inhibitor). Estane is the polymer constituent of the PBX 9501 binder. It is an amorphous, thermoplastic polyester polyurethane copolymer with a glass transition temperature $\left(\mathrm{T}_{\mathrm{g}}\right)$ of $-31{ }^{\circ} \mathrm{C}(4)$. The copolymer is composed of approximately $75 \%$ soft poly(butylene adipate) segments and $25 \%$ hard segments composed of 4,4'-methylenediphenyl 1,1'-diisocynanate and a 1,4 butanediol chain extender(5). Estane molecular weight degradation as well as the addition of the BDNPA-F plasticizer can reduce the static glass transition temperature up to 20 degrees (6), resulting in a decrease in mechanical strength and moduli.

Analyses of Estane and PBX 9501 molding powder reserves stored under uncontrolled temperature and humidity conditions indicate that the polymer molecular weight is decreasing with age as a function of hydrolysis. To mimic this aging profile the molecular weight of new Estane, in pelletized form, was degraded for 14, 23, and 36days respectively at $70^{\circ} \mathrm{C}$ and $74 \%$ RH. New Estane and the 3 degraded specimens of Estane were formulated with HMX, BDNPF-A and Irganox 1010 to produce baseline and virtually-aged PBX 9501 lots (hereafter referred to as baseline, 14, 23-, and 36-day VA PBX 9501.)

Estane weight average molecular weights $\left(\mathrm{M}_{\mathrm{w}}\right)$ were determined in tetrahydrofuran by Gel Permeation Chromatography relative to the retention times for polystyrene standards extracted from the 4 different PBX 9501 lots. $\mathrm{M}_{\mathrm{w}}$ data were measured in triplicate for each lot, with averages of $115044 \pm 691, \quad 88917 \pm 1127, \quad 72068 \pm 1389$, and $44919 \pm 793$ daltons for the baseline, 14-, 23-, and 36-day VA PBX 9501 lots respectively.
Quasi-static compression specimens, $\sim 9.5 \mathrm{~mm}$ diameter by $\sim 19.0 \mathrm{~mm}$ long, and cylindrical dogbone tensile specimens, $76.2 \mathrm{~mm}$ long with $\sim 15^{\circ}$ tapered ends, $38.1 \mathrm{~mm}$ gauge length and $12.7 \mathrm{~mm}$ diameter, were machined with the specimen loading axes parallel to the billet pressing direction. Specimen densities ranged between 1.825 to $1.831 \mathrm{~g} / \mathrm{cm}^{3}$. SHPB compression specimens were machined from PBX 9501 samples with dimensions of $6.35 \mathrm{~mm}$ diameter and $3.2 \mathrm{~mm}$ length.

\section{Low Strain Rate Testing}

Quasi-static compression tests were conducted using an Instron 5567 Materials Testing Workstation equipped with a Bemco Environmental Control Chamber at crosshead speeds of 0.5 and $5.0 \mathrm{in} / \mathrm{min}$ (strain rates approaching 0.01 and $0.1 \mathrm{~s}^{-1}$ respectively) at 3 different temperatures of $-15 \pm 1,23 \pm 1$, and $50 \pm 1^{\circ} \mathrm{C}$ with humidity ranging from $\sim 5$ to $50 \% \mathrm{RH}$. Quasi-static tensile tests were performed at the same temperatures with crosshead speeds of 1.0 and $10.0 \mathrm{in} / \mathrm{min}$ using a newly modified tensile grip design and 1 to 2 contact extensometers. The new grip design reduces the number of mechanical interfaces required to seat the specimen before uniaxial tension is achieved. Strain data was averaged if two extensometers were used. Four to five tests were completed for each test condition to evaluate specimen-to-specimen variations and all specimens were loaded to failure ( $<10 \%$ strain). Quasi-static compression specimens were also lubricated with molybdenum disulfide.

\section{High Strain Rate Compression Testing}

Two to three duplicate dynamic tests were conducted on PBX 9501 specimens at a strain rate of $\sim 2000 \mathrm{~s}^{-1}$ utilizing a modified SHPB design. Test temperatures of $-15^{\circ} \mathrm{C}$ to $+50^{\circ} \mathrm{C}$ were achieved during testing using a helium gas heating/cooling system described previously (1). Specimens were ramped to the desired temperature in approximately 5 minutes and equilibrated at temperature for approximately 10 minutes prior to testing. The SHPB sample faces were lubricated with a thin spray coating of boron nitride and a thin layer of molybdenum disulfide grease.

\section{RESULTS AND DISCUSSION}

\section{Quasi-static and Dynamic Compression Tests}

Selected compression curves are plotted in Fig. 1 for the baseline PBX 9501 lot. These data 
demonstrate a strong dependence on both strain rate and temperature. Specifically, the strength at $22^{\circ} \mathrm{C}$ increases by over a factor of 4 from $12 \mathrm{MPa}$ at $0.5 \mathrm{in} / \mathrm{min}$ to $\sim 59 \mathrm{MPa}$ at $\sim 2000 \mathrm{~s}^{-1}$. Figure 1 also shows that the peak flow stresses for the baseline PBX 9501 consistently occur at strains between $1.5 \%$ and $2.5 \%$ before slowly decaying with further strain.

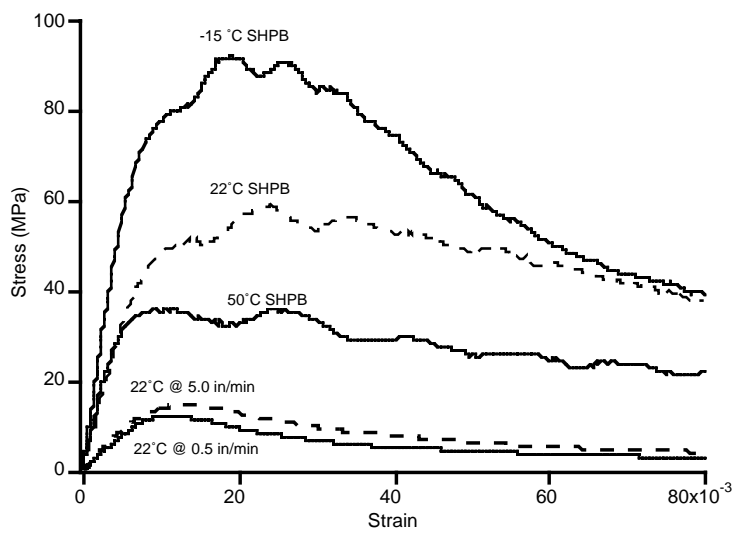

FIGURE 1. Compression behavior for baseline PBX 9501 as a function of quasi-static and dynamic strain rates and temperature.

Figures 2 and 3 are summary plots of selected compression curves for the 4 lots of PBX 9501 at quasi-static and dynamic test rates. In general the quasi-static data show a 15 to $19 \%$ increase in elastic moduli, and 18 to $23 \%$ increase in strength with an order of magnitude increase of the crosshead speed at room temperature which correlates well with previously measured PBX 9501 trends (1). Notable differences are seen in the compression strength and elastic moduli as the Estane $\mathrm{M}_{\mathrm{w}}$ is degraded from $\sim 115 \mathrm{k}$ to $\sim 45 \mathrm{k}$ with a 22 to $24 \%$ loss in strength, and between 7 and $9 \%$ loss in elastic modulus. Specifically, the 36-day VA PBX 9501 data shows compressive strengths at $5.0 \mathrm{in} / \mathrm{min}$ that are weaker than the data measured for the baseline material at the slower $0.5-\mathrm{in} / \mathrm{min}$ crosshead speed.

Conversely the effect of the degraded polymer weight is not evident in the SHPB dynamic data until the test temperature begins to approach the colder dynamic glass transition temperature $\left(T_{g}\right)$ of the soft segments. These differences suggest that the damage mechanism during high-rate loading is changing as a function of the molecular weight changes only at cold temperatures. The lack of a difference in the dynamic test data at 22 and $50^{\circ} \mathrm{C}$ as a function of molecular weight decrease, contrary to the quasi-static results, is thought to be due to the off-setting effect of the strong strain-rate sensitivity of the binder overwhelming the $M_{w}$ effect.

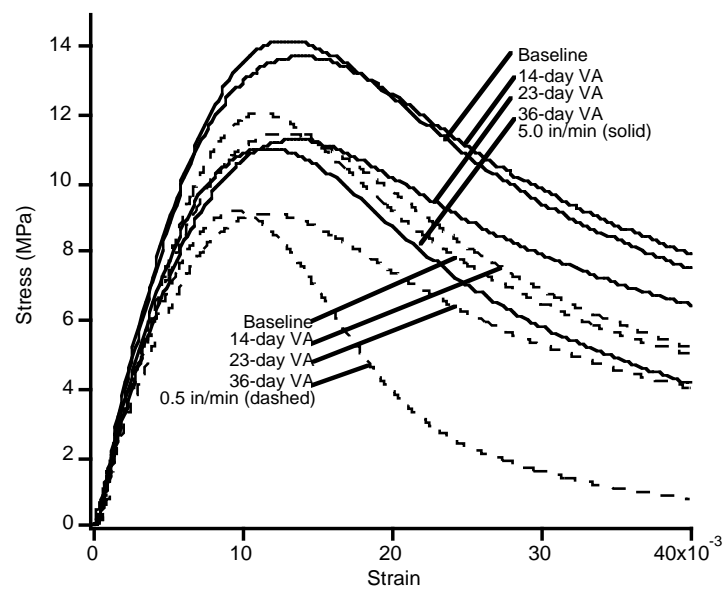

FIGURE 2. Quasi-static compression behavior for baseline and virtually-aged lots of PBX 9501 as a function of quasi-static crosshead speeds, $0.5 \mathrm{in} / \mathrm{min}$ (dashed) and $5.0-\mathrm{in} / \mathrm{min}$ (solid).

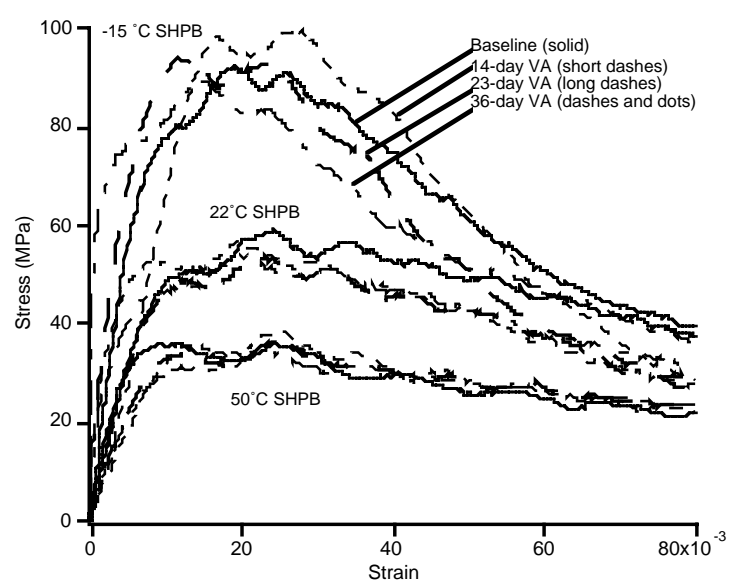

FIGURE 3. SHPB compression behavior for baseline and virtually-aged lots of PBX 9501 as a function of temperature at dynamic strain rates.

\section{Quasi-static Tensile Tests}

Figure 4 is a plot of the stress strain curves for several of the baseline PBX 9501 specimens at the three different test temperatures and two different crosshead speeds where the test data show good reproducibility from specimen-to-specimen. In general the tensile behavior exhibits rate and 
temperature dependence similar to the compressive behavior, but with peak flow stresses, and the strain at which the peak flow stress significantly decreased. A drop in the peak flow stress was also evident at $-15^{\circ} \mathrm{C}$ when the quasi-static crosshead speed was increased by an order of magnitude because of increasingly brittle behavior.

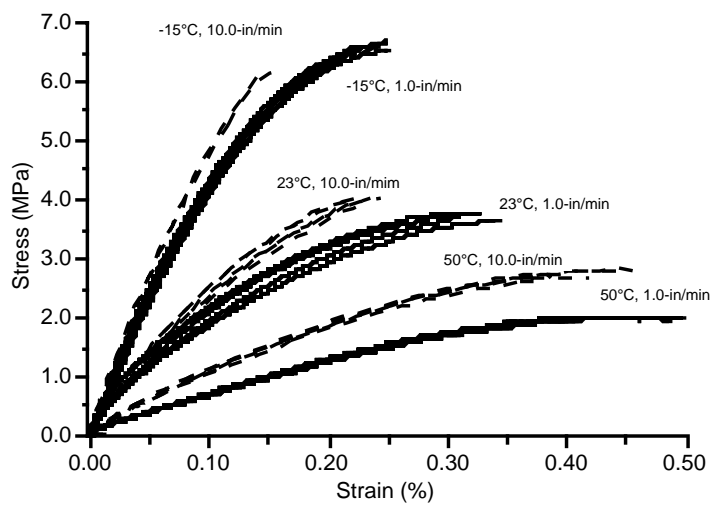

FIGURE 4. Tensile behavior of baseline PBX 9501 at 2 crosshead speeds, 1.0- and 10-in/min, and three temperatures $\left(-15,23\right.$, and $\left.50^{\circ} \mathrm{C}\right)$.

Figure 5 is a summary plot of selected tensile curves at $22^{\circ} \mathrm{C}$ and 1.0 -in/min for all $4 \mathrm{PBX} 9501$ lots (baseline and VA). Significant differences in the tensile response due to virtual aging are not immediately evident in these data until the $M_{w}$ is degraded by more than $37 \%$. These trends were also evident at the other two test temperatures, -15 and $50{ }^{\circ} \mathrm{C}$. Similar to the baseline data, the peak flow stress at $-15^{\circ} \mathrm{C}$ for the three virtually-aged lots also drop to a lower value at the fast crosshead speed.

\section{SUMMARY AND CONCLUSIONS}

The following conclusions can be drawn: 1) the compressive and tensile stress-strain response of PBX 9501 demonstrates strong strain rate and temperature dependence; 2) Estane molecular weight degradation significantly affects the quasi-static compressive behavior at all temperatures, 3) the dynamic test data at $-15^{\circ} \mathrm{C}$ exhibits significant changes as a function of the molecular weight, suggesting changes in the failure mechanism, while no effect of $M_{w}$ was seen dynamically at 22 or $50^{\circ} \mathrm{C}$; and 4) the tensile response is considerably weaker and has lower flow stress with smaller differences evident in the strength, elastic moduli and flow stress as a function of molecular weight changes in comparison to compression data.

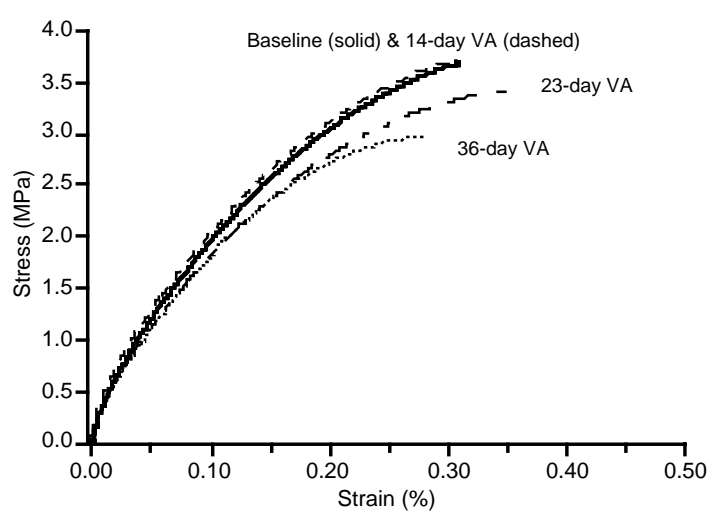

FIGURE 5. Tensile behavior of baseline, 14-, 23- and 36-day virtually-aged $\mathrm{PBX} 9501$ at $\sim 23^{\circ} \mathrm{C}, 1.0-\mathrm{in} / \mathrm{min}$.

\section{ACKNOWLEDGMENTS}

The authors wish to thank Debra Wrobleski, Bruce Orler, Sheldon Larson, Ray Flesner, Gregg Sullivan, Robert Montoya, George Harper, Jose Archueta, and Wayne King for the preparation, density, and $M_{w}$ characterization of the degraded Estane and PBX 9501 materials. This work was performed under the auspices of the U.S. Department of Energy (contract W-7405-ENG-36).

\section{REFERENCES}

1. Gray III, G. T., Blumenthal, W. R., Idar, D. J. and Cady, C. M., "Influence of Temperature on the HighStrain-Rate Mechanical Behavior of PBX 9501," in Shock Compression of Condensed Matter-1997, edited by S.C. Schmidt et al., AIP Conference Proceedings 429, New York, 1998, pp. 583-586.

2. Idar, D. J., Rabie, R. L., and Scott, P. D., "Quasi-Static, Low-Strain Rate Compression Measurements of Thermally Treated and Mechanically Insulted PBX 9502 Samples," Los Alamos National Laboratory report LA-UR-97-5116, Los Alamos, New Mexico, (1997).

3. Idar, D.J. and Holmes, M.D. "Quasi-static, Low Strain Rate Compression Measurements of PBX 9502 and Mock 900-24 Specimens," Los Alamos National Laboratory report LA-UR-98-5270, Los Alamos, New Mexico, (1998).

4. Goodrich, B. F., "Adhesives Technology of Estane Polyurethane", B.F. Goodrich Speciality Chemicals, TSR 76-02 TF116, March 1995.

5. Hoffman, D.M., Caley, L.E. ACS Div. Org. Coat. Plast. Chem. 44, (1981) p. 686.

6. Campbell, M.S., Garcia, D.A., Idar, D.J., "Effects of Temperature and Pressure on the Glass Transitions of Plastic Bonded Explosives", Thermochimica Acta, 357-358, (2000) pp. 89-95. 